\title{
Research on Personalized Community E-Learning Recommendation Service System by Using Adaptive Filtering Algorithm
}

\author{
Liang Zhao ${ }^{1}$ and Qi Luo ${ }^{2,3}$ \\ ${ }^{1}$ School of Mechanical and Electronic Engineering, Wuhan University of Technology, \\ Wuhan 430070, China \\ ${ }^{2}$ Information Engineering school, \\ Wuhan University of Science and Technology and Zhongnan Branch, \\ Wuhan 430223, China \\ ${ }^{3}$ College of Engineering and Technology, Southwest University, \\ Chongqing 400715, China \\ Hejm2000@163.com, Ccnu_luo2008@yahoo.com.cn
}

\begin{abstract}
To meet the needs of education in the learning community, an adaptive filtering algorithm for teaching resources based on vector space model was proposed in the paper. First, feature selection and pseudo feedback were used to select the initial filtering profiles and thresholds through training algorithm. Then user feedback was utilized to modify the profiles and thresholds adaptively through filtering algorithm. The algorithm had two advantages, the first was that it could carry on self-study to improve the precision; the second was that the execution did not need massive initial texts in the process of filtering. The result manifested that the algorithm was effective.
\end{abstract}

Keywords: Adaptive Filtering Algorithm, Vector Space Model, Personalized Community E-learning Recommendation Service.

\section{Introduction}

With the increase of computers and network, people pay more and more attention in community E-learning. E-learning can break through the limit of space and time, reduce learning cost and improve learning efficiency. Therefore, many community websites based on E-learning have also been constructed; community residents can get some information or some study courses. But the application of these websites is difficult to attract community resi-dents' initiative participation [1]. The investigation indicates that personalized recommendation service system is imperfect. If the community E-learning wants to attract residents, the idea of personalized design should be needed. It means that the personalized knowledge and information service should be recommended according to residents' needs.

At present, many scholars have carried on a great deal of researches on filtering algorithms, such as traditional batch filtering algorithm [2].But those algorithms have some disadvantages, For example, it needs massive initial training texts, yet the preci- 
sion and the recall is low [3]. According to this, an adaptive filtering algorithm for teaching resources based on vector space model is proposed in the paper. It has improved the traditional algorithm and introduced adaptive feedback study mechanism in intelligence control. The advantage of the algorithm is that it can carry on selfstudy to improve the precision, and the execution does not need massive initial texts in the process of filtering.

\section{Adaptive Filtering Algorithm}

Adaptive filtering algorithm based on vector space model is composed of two steps.

Step1: training phase. The task of training stage is to get the initial filtering profile and set the initial threshold.

Step2: adaptive filtering phase. In adaptive filtering, the main task is to modify the profile and threshold adaptively.

\subsection{Training Stage}

Fig.1 shows the architecture of the training stage. At first, feature vectors are extracted from positive and pseudo-positive documents. The pseudo-positive documents are those that have high similarity with the topic but haven't been labeled as positive documents in the training set. The pseudo-positive documents can be obtained by several ways. We can get pseudo-positive documents by pseudo feedback. We also can get the pseudo-positive documents by using the hierarchy of categories: a topic's pseudo-positive documents are those that have the same high-level categories provided by the training set.

To get the feature vectors, we first remove stop-words and do morphological analysis on remaining words. Then, we compute the logarithm mutual information between words and topics [4]. The formula 1 is shown as follows:

$$
\log M I\left(w_{i} \cdot T_{j}\right)=\log \left(\frac{p\left(w_{i} / T_{j}\right)}{p\left(w_{i}\right)}\right)
$$

Where $w_{i}$ is the $\mathrm{i}$-th word and $T_{j}$ is the $\mathrm{j}$-th topic. Higher logarithm mutual information means $w_{i}$ and $T_{j}$ are more relevant. $p\left(w_{i} / T_{j}\right)$ and $p\left(w_{i}\right)$ are estimated by maximal likelihood method.

After gotten the feature vectors of positive and pseudo-positive documents, we merged them into the initial profile. The initial profile is the weighted sum of positive and pseudo-positive feature vectors. Then we should set initial threshold for each topic. The initial threshold is set based on the similarity of each document in the training set. The similarity between a profile and training document is computed by the cosine formula 2 .

$$
\operatorname{Sim}\left(d_{i}, p_{j}\right)=\cos \theta=\frac{\sum_{k} d_{i k} p_{j k}}{\sqrt{\sum_{k} d_{i k}^{2} \sum_{k} p_{j k}^{2}}}
$$


Where $p_{j}$ is the profile vector of the $\mathrm{j}$-th topic and $d_{i}$ is the vector representation of the i-th document. $d_{i k}$ is the weight of the k-th word in $d_{i} \cdot d_{i k}$ is computed as formula 3 .

$$
d_{i k}=1+\log \left(t f_{i k} a v d l / d l\right)
$$

Where $t f_{i k}$ is the term frequency of the k-th word in the i-th document, the $d l$ is the document length counted by tokens in the document after morphological processing and stop-words removal, the $a v d l$ is the average document length gotten from training set. According to the similarities of training documents, each initial threshold is set to get the best filtering performance.

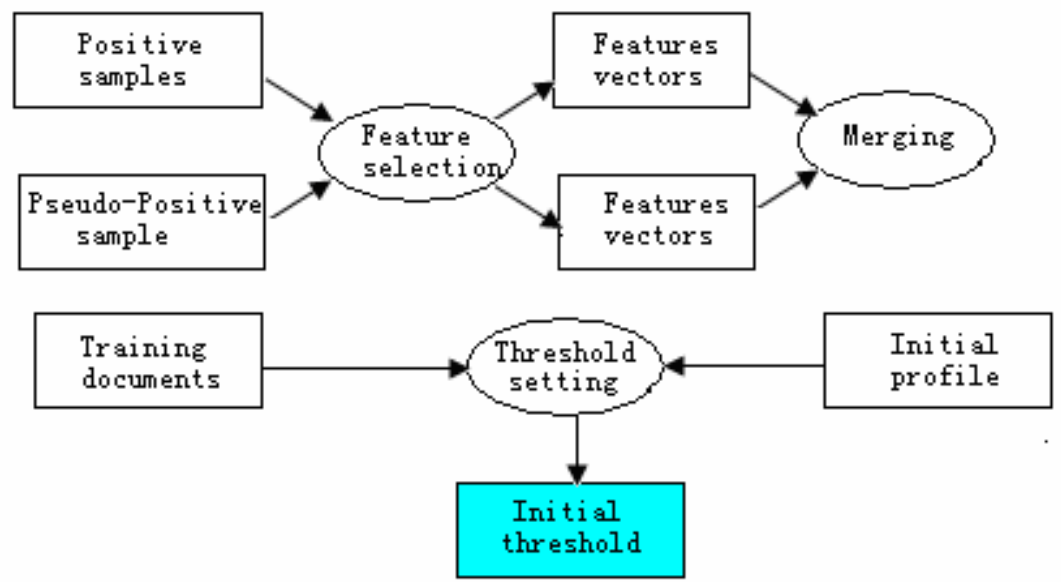

Fig. 1. Architecture of the training stage

\subsection{Adaptive Filtering Architecture}

Fig. 2 shows the architecture of adaptive filtering. When a document arrives, its similarity with the topic profile is calculated. If its similarity is higher than the current threshold, then this document is retrieved and the user's relevance judgment is gotten.

If the document is really relevant to the topic, it will be considered as positive sample, otherwise negative sample. The vectors of positive and negative samples will be used to modify the topic's profile, which is shown in formula 4.

$$
p_{j}^{\prime}=p_{j}+\alpha p_{j}(p o s)+\beta p_{j}(\text { neg })
$$

Where $p_{j}^{\prime}$ is the topic's profile after modification, $p_{j}$ is the topic's profile before modification, $p_{j}(\cos )$ is vector of positive samples gotten at this updating interval while $p_{j}(n e g)$ is vector of negative samples; $\alpha$ and $\beta$ are the weight of positive and negative vectors respectively. 


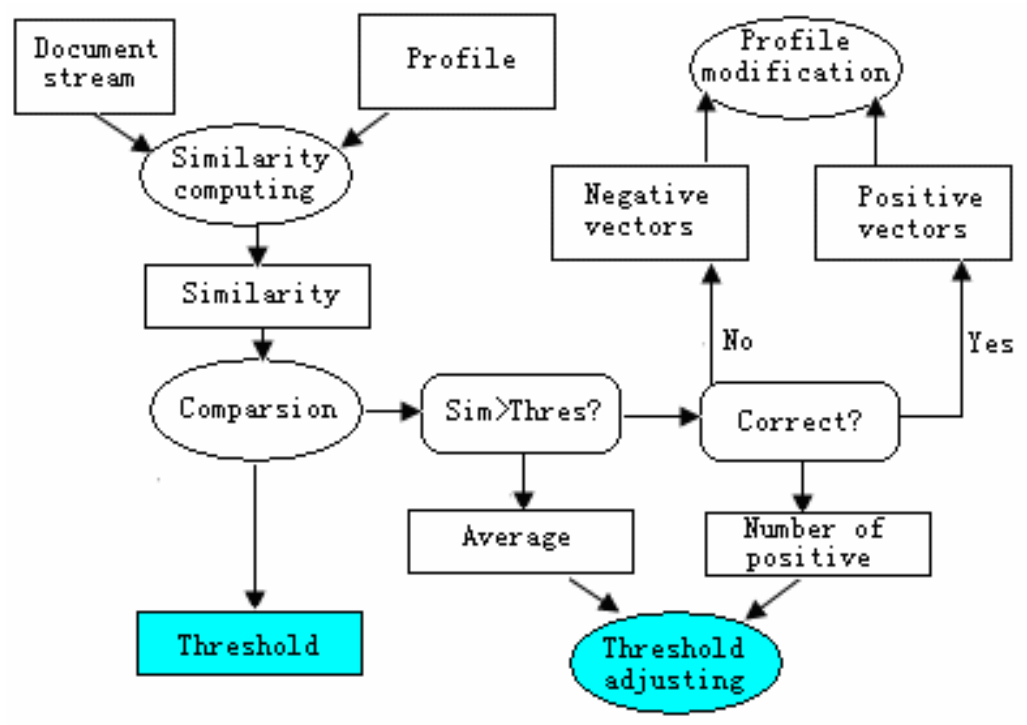

Fig. 2. Architecture of the adaptive filtering

\section{Conclusion}

In summary, a new adaptive filtering algorithm for community E-learning based on vector space model is proposed in the paper. The results manifest that the algorithm is effective through testing in personalized recommendation service system based on community E-learning.

\section{References}

1. Yanwen W. and Zhonghong W.: Knowledge Adaptive Presentation Strategy in E-Learning. Proceedings of Second International Conference on Knowledge Economy and Development of Science and Technology, Beijing, 2004, 6-9.

2. Lawrence R D, Almasi G S, Kotlyar V, et al.: Personalization of Supermarket Product Recommendations. Special Issue of the International Journal Data Mining and Knowledge Discovery, Vol.5, 2001, 11-32.

3. Nie Xin.: Take about the Digital Individualized Information Service of Library. Information Science Journal, vol.23, 2005, 1-5.

4. Robertson S and Hull DA.: The TREC-9 filtering track final report. Proceedings of the 9th Text Retrieval Conference. Gaithersburg, 2001, 25-40. 